\title{
On finiteness of chains of intermediate rings
}

\author{
Mabrouk Ben Nasr
}

Received: 2 March 2008 / Accepted: 2 December 2008 / Published online: 8 January 2009

C The Author(s) 2008. This article is published with open access at Springerlink.com

\begin{abstract}
An extension of integral domains $R \subseteq S$ is said to have the "finite length of intermediate chains of domains" property (for short FICP) if each chain of intermediate rings between $R$ and $S$ is finite. The main purpose of this paper is to characterize when $R \subseteq S$ has FICP in case $R^{*}$ (the integral closure of $R$ in $S$ ) is a finite dimensional semilocal domain. This generalizes a theorem due to Gilmer, in which $S$ is the quotient field of $R$. Examples illustrating the sharpness and the limits of our results are settled.
\end{abstract}

Keywords Ring extension - Intermediate chains of domains - Normal pair . Valuation domain

Mathematics Subject Classification (2000) Primary 13B02 - 13B22 · 13F05 . 13G05

\section{Introduction}

All rings considered below are integral, commutative with identity; and all subrings are unital. If $R$ is an integral domain, let $q f(R)$ denote the quotient field of $R$ and $R^{\prime}$ the integral closure of $R$. If $R \subseteq S$ is a ring extension, denote by $R^{*}$ the integral closure of $R$ in $S$ and by $[R, S]$ the set of all rings $T$ such that $R \subseteq T \subseteq S$.

A frequent theme in the study of intermediate rings between $R$ and $S$ is the finiteness of the length of chains of intermediate rings between $R$ and $S$ (cf. [1,5,6]). This

Communicated by D. Segal.

M. B. Nasr $(\varangle)$

Department of Mathematics, Faculty of Sciences of Sfax, BP 802, 3018 Sfax, Tunisia

e-mail: mabrouk_bennasr@yahoo.fr 
property is labeled as (FICP): "finite length of intermediate chains of domains" property. Our work is motivated by two papers. The first is [6] in which Jaballah studied the finiteness of length of chains in $[R, S]$ when $(R, S)$ is a normal pair. Our second motivation is [5], in which Gilmer both developed several techniques useful for studying FICP in $[R, q f(R)]$ and derived characterizations of such domains. The main result in this paper generalizes the work of Gilmer [5] who showed, among other things, that if $R$ is an integral domain with quotient field $K$, then $R \subseteq K$ has FICP iff $R \subseteq R^{\prime}$ and $R^{\prime} \subseteq K$ have FICP.

As the title of this paper suggests, our goal is to obtain a necessary and sufficient condition for the length of chains of intermediate rings to be finite. Precisely, Theorem 2.4 states that if $R \subseteq S$ is a ring extension such that $R^{*}$ is a semilocal domain with finite Krull dimension, then $R \subseteq S$ has FICP iff each of the extensions $R \subseteq R^{*}$ and $R^{*} \subseteq S$ has FICP. This generalizes Gilmer's study, in which $S=q f(R)$.

In addition to the above notations and conventions, denote by $\operatorname{Spec}(R)$ (resp., $\operatorname{Max}(R))$ the set of prime (resp., maximal) ideals of $R . P \subseteq Q$ are two prime ideals of $R$, let $[P, Q]$ denote the set of all prime ideals $Q^{\prime}$ of $R$ such that $P \subseteq Q^{\prime} \subseteq Q$. As usual, $\subset$ denotes proper inclusion. Any unexplained terminology is standard as in [7].

\section{Main results}

As an initial step toward understanding what extensions $R \subseteq S$ have FICP, we will show that this class is closed under localization and factor domains. The simple proof of this result is included for the sake of completeness.

Proposition 2.1 Let $R \subseteq S$ be a ring extension. Assume that $R \subseteq S$ has FICP, then the following hold:

(i) For each multiplicative closed subset $N$ of $R, N^{-1} R \subseteq N^{-1} S$ has FICP.

(ii) For each $Q \in \operatorname{Spec}(S)$, setting $P=Q \cap R$ then $R / P \subseteq S / Q$ has FICP.

Proof (i) and (ii). Let $N$ be a multiplicative subset of $R$. For a prime ideal $Q$ of $S$ with contraction $P$ in $R$, let $\varphi: S \rightarrow S / Q$ the canonical surjection. If $T_{1} \in\left[N^{-1} R, N^{-1} S\right]$ (resp., $[R / P, S / Q]$ ), then $T_{1} \cap S$ (resp., $\left.\varphi^{-1}\left(T_{1}\right)\right) \in[R, S]$ and a straightforward calculation shows that $T_{1}=N^{-1}\left(T_{1} \cap S\right)$ (resp., $\left.T_{1}=\varphi^{-1}\left(T_{1}\right) / Q\right)$. Hence, the assignment $T_{1} \mapsto T_{1} \cap S$ (resp., $\left.T_{1} \mapsto \varphi^{-1}\left(T_{1}\right)\right)$ gives an increasing injection from $\left[N^{-1} R, N^{-1} S\right]$ (resp., $[R / P, S / Q]$ ) to $[R, S]$. As the latter extension has FICP, so is the former.

In view of Proposition 2.1(i), it is natural to ask whether "locally FICP" implies FICP. The following example answers the question in the negative, while giving a positive answer if the basic ring is semilocal domain (see Proposition 2.3).

We recall that if $R \subset S$ is a pair of rings and $P$ is a prime ideal of $R$, then we mean by $S_{P}$ the ring $S_{N}$ where $N=R-P$.

Example 2.2 Let $R$ be a one dimensional Prüfer domain with infinitely many maximal ideals and take $S=q f(R)$. Notice that, for each maximal ideal $M$ of $R$ each chain of rings between $R_{M}$ and $S_{M}$ has length at most 2 . On the other hand, let $\left\{M_{i}, i \geq 0\right\}$ be a subset of maximal ideals of $R$ and take $T_{i}=\cap_{0 \leq k \leq i} R_{M_{k}}$. Then $T_{0} \supset T_{1} \supset \ldots$ is a strictly descending chain of intermediate rings between $R$ and $S$. 
Proposition 2.3 Let $R$ be an integral domain such that Max $(R)$ is finite. Then $R \subseteq S$ has FICP if and only if $R_{M} \subseteq S_{M}$ has FICP for $M \in \operatorname{Max}(R)$.

Proof If $R \subseteq S$ satisfies FICP, then so is $R_{M} \subseteq S_{M}$ for each maximal ideal $M$ of $R$ [Proposition 2.1(i)]. Conversely, suppose $\operatorname{Max}(R)=\left\{M_{1}, \ldots, M_{n}\right\}$ and assume that $R_{M_{i}} \subseteq S_{M_{i}}$ has FICP for each $i$. Because $T=\cap_{1 \leq i \leq n} T_{M_{i}}$ for each $T \in[R, S]$, it follows that $T=T_{1} \cap \ldots \cap T_{n}$ where $T_{i} \in\left[R_{M_{i}}, S_{M_{i}}\right]$. Hence $[R, S]$ has FICP, as was to be proved.

Before presenting our main result, we recall that if $R \subseteq S$ is a ring extension then $(R, S)$ is said to be a residually algebraic pair if for any $\operatorname{ring} T$ in $[R, S]$ and any prime $Q$ of $T, T / Q$ is algebraic over $R /(Q \cap R)$ [2, Definitions 1.1 and 2.1]. The pair $(R, S)$ is said to be normal if each $T \in[R, S]$ is integrally closed in $S$ [4]. Moreover it was shown in [2, Theorem 2.10] that $(R, S)$ is a normal pair iff it is residually algebraic and $R$ is integrally closed in $S$.

The proof of Theorem 2.4 depends ultimately on normal pairs techniques. So it is convenient to recall some useful facts about these pairs [2, Theorem 2.5(v), Lemma 3.1(iii) and Lemma 2.9(i)]. Let $(R, S)$ be a normal pair. Set $\operatorname{Max}(R)=\left\{M_{i} \mid i \in I\right\}$, then for each $i \in I$ there exists a prime ideal $Q_{i}$ of $R$ such that $Q_{i} \subseteq M_{i}, S_{M_{i}}=R_{Q_{i}}$ and $R_{M_{i}} / Q_{M_{i}}$ is a valuation domain. Moreover for each $T \in[R, S]$ and for each prime ideal $Q$ of $T$, set $P=Q \cap R$, then $T_{Q}=R_{P}$.

We now present the titular result which is the most important of our paper, as it constitute our main generalization of Gilmer's result [5, Theorem 2.3] who proved, among other things, that if $R$ is an integral domain with quotient field $K$, then $R \subseteq K$ has FICP (in the terminology of Gilmer, $R$ is an FC-domain) iff $R \subseteq R^{\prime}$ and $R^{\prime} \subseteq K$ have FICP.

Theorem 2.4 Let $R \subseteq S$ be a ring extension such that $R^{*}$ is semilocal with finite Krull dimension. Then $R \subseteq S$ has FICP if and only if each of the extensions $R \subseteq R^{*}$ and $R^{*} \subseteq S$ has FICP.

The proof of this theorem breaks into two lemmas.

Lemma 2.5 Let $(R, S)$ be a normal pair. Set $\operatorname{Max}(R)=\left\{M_{i} \mid i \in I\right\}$ and for each $i \in I$, let $Q_{i}$ the prime ideal of $R$ such that $S_{M_{i}}=R_{Q_{i}}$. For a ring $T \in[R, S]$ and a maximal ideal $Q$ of $T$, there exists $i \in I$ such that $Q \cap R \in\left[Q_{i}, M_{i}\right]$.

Proof Let $T \in[R, S]$ and $Q \in \operatorname{Max}(T)$. Set $P=Q \cap R$. There exists a maximal ideal $M_{i}$ of $R$ such that $P \subseteq M_{i}$. Let $P_{i}$ (resp., $Q_{i}$ ) be the prime ideal of $R$ such that $T_{M_{i}}=R_{P_{i}}$ (resp., $S_{M_{i}}=R_{Q_{i}}$ ). Such ideals $P_{i}$ and $Q_{i}$ exist since $(R, T)$ and $(R, S)$ are normal pairs. As $Q T_{M_{i}} \in \operatorname{Max}\left(T_{M_{i}}\right)$, then $Q T_{M_{i}}=P_{i} R_{P_{i}}$. Thus $P=P_{i}$. Now since $R_{M_{i}} \subseteq T_{M_{i}} \subseteq S_{M_{i}}$, then we obtain $P_{i}=P \in\left[Q_{i}, M_{i}\right]$, the desired conclusion.

Lemma 2.6 Let $R \subseteq S$ be a ring extension. Suppose that $R^{*} \subseteq S$ has FICP. Then:

(i) $(R, S)$ is a residually algebraic pair.

(ii) For each $T \in[R, S]$, let $J=T \cap R^{*}$. Then $(J, T)$ is a normal pair. 
Proof (i) To prove that $(R, S)$ is a residually algebraic pair, it is enough to show that $\left(R^{*}, S\right)$ is a residually algebraic pair [2, Remark 2.2]. For this end, let $T \in\left[R^{*}, S\right]$ and $Q \in \operatorname{Spec}(T)$. Set $P=Q \cap R^{*}$. Since $R^{*} \subseteq S$ has FICP then so is $R^{*} \subseteq T$. According to Proposition 2.1(ii) $R^{*} / P \subseteq T / Q$ has also FICP. Assume by way of contradiction that $R^{*} / P \subseteq T / Q$ is not algebraic. Then there exists an element $t$ of $T / Q$ transcendental over $R^{*} / P$. Hence, we obtain $\left(R^{*} / P\right)[t] \supset\left(R^{*} / P\right)\left[t^{2}\right] \supset \ldots$ a strictly descending chain of intermediate rings between $R^{*} / P$ and $T / Q$, a contradiction.

(ii) Since $[J, T] \subseteq[R, S]$ and $(R, S)$ is a residually algebraic pair (by assertion (i)), then $[J, T]$ inherits the "residually algebraic pair" property from $[R, S]$. Now our task is to show that $J$ is integrally closed in $T$. Indeed, let $x \in T$ such that $x$ is integral over $J$, then $x$ is integral over $R$ and hence $x \in T \cap R^{*}=J$. The desired conclusion.

Proof of Theorem 2.4 It is clear that the stated condition is necessary. For sufficiency, assume that $R \subseteq R^{*}$ and $R^{*} \subseteq S$ have FICP. Set $\operatorname{Max}\left(R^{*}\right)=\left\{M_{k}, 1 \leq k \leq n\right\}$. For each $1 \leq k \leq n$ there exists a prime ideal $Q_{k}$ of $R^{*}$ such that $Q_{k} \subseteq M_{k}$ and $S_{M_{k}}=R^{*} Q_{k}$. Now, let $T_{0}=R \subseteq T_{1} \subseteq \cdots \subseteq S$ be a chain of intermediate rings in $[R, S]$. Set $\operatorname{Max}\left(T_{i}\right)=\left\{m_{i j}, j \in I_{i}\right\}, J_{i}=T_{i} \cap R^{*}$ for each $i$ and let $p_{i j}=m_{i j} \cap J_{i}, j \in I_{i}$. By Lemma 2.6(ii), we have shown that $\left(J_{i}, T_{i}\right)$ is a normal pair for each $i$, so that $\left(T_{i}\right)_{m_{i j}}=\left(J_{i}\right)_{p_{i j}}$ and $T_{i}=\cap_{j \in I_{i}}\left(J_{i}\right)_{p_{i j}}$. Because $R \subseteq R^{*}$ has FICP, the considered chain has the following finite contraction, say: $J_{0}=R \subseteq J_{1} \subseteq \cdots \subseteq J_{S}=R^{*}$. Hence, to show that $R \subseteq S$ has FICP it suffices to show that the set $\mathcal{F}=\left\{p_{i j} \mid j \in I_{i}, 1 \leq i \leq s\right\}$ is finite. Denote by $T_{i}^{*}$ the integral closure of $T_{i}$ in $S$. Of course $T_{i} \subseteq T_{i}^{*}$ is an integral extension, so there exists a maximal ideal $m_{i j}{ }^{*}$ in $T_{i}^{*}$ lying over $m_{i j}$. Set $p_{i j}{ }^{*}=m_{i j}{ }^{*} \cap R^{*}$, notice that $\left(R^{*}, S\right)$ is a normal pair (Lemma 2.6), then by Lemma 2.5, $p_{i j}{ }^{*} \in\left[Q_{k}, M_{k}\right]$ for some $1 \leq k \leq n$. On the other hand, $R_{M_{k}}^{*} /\left(Q_{k}\right)_{M_{k}}$ is a valuation domain with finite Krull dimension, then $\left[Q_{k}, M_{k}\right]$ is a totally ordered set which is finite for each $1 \leq k \leq n$. Finally, notice that $p_{i j}=p_{i j}{ }^{*} \cap J_{i}$. Thus, $\mathcal{F} \subseteq \cup_{1 \leq i \leq s} \cup_{1 \leq k \leq n}\left[Q_{k} \cap J_{i}, M_{k} \cap J_{i}\right]$. Therefore, $\mathcal{F}$ is finite, and this completes the proof.

In [5], Gilmer defined the term "FC-domain" which means an integral domain $R$ such that $[R, q f(R)]$ has FICP.

As a consequence of Theorem 2.4, we recover Gilmer's result.

Corollary 2.7 [5, Theorem 3.1] Let $R$ be an integral domain with quotient field $K$. Then $R$ is an FC-domain if and only if each of the extensions $R \subseteq R^{\prime}$ and $R^{\prime} \subseteq K$ has FICP.

In view of Theorem 2.4, one asks whether "FICP" is transitive. The next example provides extensions of domains $R \subseteq T \subseteq S$ such that both of $R \subseteq T$ and $T \subseteq S$ have FICP, while $R \subseteq S$ does not have PICP.

Example 2.8 Let $R=\mathbb{Z}_{(2)}, T=q f(R)=\mathbb{Q}$ and $S=\mathbb{Q}(i)$, where $i$ is the complex number such that $i^{2}=-1$. The extensions $R \subseteq T$ and $T \subseteq S$ have FICP since $R$ is a valuation domain with finite spectrum and $[S: T]<+\infty$. On the other hand, 
suppose that $R \subseteq S$ has FICP and consider $T_{k}=\mathbb{Z}_{(2)}+2^{k} i \mathbb{Z}_{(2)}$ for each positive integer $k$. It is clear that $T_{k} \subseteq T_{k+1}$ and $T_{k} \in[R, S]$ for each $k$. Hence, there exist positive integers $k \neq l$ such that $T_{k}=T_{l}$. In particular, there exist $a, b \in \mathbb{Z}_{(2)}$ such that $2^{k} i=a+b 2^{l} i$, a contradiction.

We close this section by an application of our results to a well known class of domains, namely pullbacks. Let $T$ be an integral domain, $I$ an ideal of $T$ and $D$ a subring of $T / I$. Consider the pullback construction:

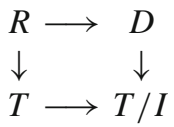

Following [3], we say that $R$ is the domain of the $(T, I, D)$ construction, and we set $R:=(T, I, D)$.

Notice that there is a one-to-one correspondence between $[R, T]$ and $[R / I, T / I]$. Then $R \subseteq T$ has FICP iff $D \subseteq T / I$ has FICP. Thus we have:

Corollary 2.9 Let $R:=(T, M, D)$ such that $M$ is a maximal ideal of $T$ and $D$ is a domain with quotient field $K=T / M$. Then the extension $R \subseteq T$ has FICP iff $D^{\prime}$ is a Prïfer domain with finite spectrum and $D \subseteq D^{\prime}$ has FICP.

Proof Follows from Corollary 2.7 and [5, Theorem 1.5].

We next state a meaningful upshot of Lemma 2.6.

Corollary 2.10 Let $R:=(T, M, k)$ such that $M$ is a maximal ideal of $T$ and $k$ is a subfield of the field $K=T / M$. Then $R \subseteq T$ has FICP iff $[K: k]<+\infty$.

Proof It remains to prove that $k \subseteq K$ has FICP iff $k \subseteq K$ is a finite field extension. The "only if" half is trivial. For the "if" half it follows readily from Lemma 2.6(i) that $k \subseteq K$ is an algebraic extension. Hence, it will suffice to show that $K$ is a finite $k$-module. By way of contradiction, we seek $t_{1} \in K \backslash k$ such that $k\left[t_{1}\right] \neq K$. Inductively we can find $\left(t_{n}\right)_{n \geq 2}$ a sequence of elements of $K$ such that $t_{n+1} \in K \backslash k\left[t_{1}, \ldots, t_{n}\right]$ for each integer $n \geq 2$. This gives rise to the following strictly ascending chain of rings between $k$ and $K$

$$
k \subset k\left[t_{1}\right] \subset \cdots \subset k\left[t_{1}, \ldots, t_{n}\right] \subset \cdots \subset K,
$$

contradicting that $k \subseteq K$ has FICP.

Despite the motivating result [5], we have seen that $R \subseteq q f(R)$ has FICP iff $R \subseteq R^{\prime}$ and $R^{\prime} \subseteq q f(R)$ satisfy FICP iff $R \subseteq R^{\prime}$ has FICP and $R^{\prime}$ is a Prüfer domain with finite spectrum. In what follows we provide an example of an extension $R \subseteq S$ such that $R \subseteq S$ has FICP but $R^{*}$ is not a Prüfer domain. This example depends ultimately on the pullback techniques. 
Example 2.11 Let $k \subseteq K$ be an extension of fields such that $[K: k]<+\infty$ and let $X, Y$ two indeterminates over $k$. Set $m=(X, Y) K[X, Y], n=(X+1) K[X, Y]$, $U=K[X, Y] \backslash\{m \cup n\}$ and $T=U^{-1} K[X, Y]$. Then $T$ is not a Prüfer domain. Moreover, $T$ is semi local with two maximal ideals $M=U^{-1} m$ and $N=U^{-1} n$ and $\operatorname{dim}$ $(T)=2$. Now, let $R=(T, M, k)$ and $S=T_{M}$. One check easily that $[T, S]=\{T, S\}$. Then $T \subseteq S$ has FICP. On the other hand, since $[K: k]<+\infty$, then by Corollary 2.10 $R \subseteq T$ has FICP. Finally, we prove that $R \subseteq S$ has FICP. By Theorem 2.4, it suffices to show that $R^{*}=T$. It is clear that $R^{*} \subseteq T^{\prime}=T$ since $T$ is integrally closed. Also, we have $k \subseteq K$ is an algebraic extension since [ $K: k]<+\infty$. An application of [3, Lemme 2], shows that $R \subseteq T$ is an integral extension which implies $T \subseteq R^{*}$. Thus $R^{*}=T$, as we wished to show.

Open Access This article is distributed under the terms of the Creative Commons Attribution Noncommercial License which permits any noncommercial use, distribution, and reproduction in any medium, provided the original author(s) and source are credited.

\section{References}

1. Anderson, D.D., Dobbs, D.E., Mullins, B.: The primitive element theorem for commutative algebras. Houston. J. Math. 25, 603-623 (1999)

2. Ayache, A., Jaballah, A.: Residually algebraic pairs of rings. Math. Z. 225, 49-65 (1995)

3. Cahen, P.-J.: Couples d'anneaux partageant un idéal. Arch. Math. 51, 505-514 (1988)

4. Davis, E.D.: Overrings of commutative rings III: Normal pairs. Trans. A. M. S 182, 1175-1185 (1973)

5. Gilmer, R.: Some finiteness conditions on the set of overrings of an integral domain. Proc. Am. Math. Soc. 131(8), 2337-2346 (2003)

6. Jaballah, A.: Finiteness of the set of intermediary rings in normal pairs. Saitama Math. J. 17, 59-61 (1999)

7. Kaplansky, I.: Commutative Rings, 2nd edn. University of Chicago Press, Chicago (1974) 